Portland State University

PDXScholar

Physics Faculty Publications and Presentations

Physics

$9-1-1981$

\title{
Time-Dependent Direct Correlation Function
}

John D. Ramshaw

Portland State University, jdramshaw@yahoo.com

Follow this and additional works at: https://pdxscholar.library.pdx.edu/phy_fac

Part of the Physics Commons

Let us know how access to this document benefits you.

\section{Citation Details}

J.D. Ramshaw, "Time-dependent direct correlation function," Phys. Rev. A 24, 1567 (1981)

This Article is brought to you for free and open access. It has been accepted for inclusion in Physics Faculty Publications and Presentations by an authorized administrator of PDXScholar. Please contact us if we can make this document more accessible: pdxscholar@pdx.edu. 


\title{
Time-dependent direct correlation function
}

\author{
John D. Ramshaw \\ Theoretical Division, University of California, Los Alamos National Laboratory, Los Alamos, New Mexico 87545 \\ (Received 23 March 1981)
}

\begin{abstract}
A time-dependent direct correlation function (TDDCF) is defined and discussed. The definition (which differs from that proposed by Percus and Yevick) is motivated by linear response theory. The TDDCF consequently has a functional-derivative or stimulus-response interpretation closely analogous to that of the static direct correlation function. Mean-field considerations suggest that the TDDCF separates into a term which is simply related to the pair potential and a spatially shorter-ranged remainder. This behavior is confirmed in the Vlasov-Zwanzig approximation.
\end{abstract}

\section{INTRODUCTION}

The direct correlation function (DCF) has proved to be a very fruitful concept in terms of which to discuss the equilibrium properties of atomic and molecular fluids..$^{1-4}$ It therefore seems worthwhile to consider whether the DCF admits a natural generalization to correlations in time as well as space. Percus and Yevick ${ }^{5}$ proposed one such generalization, which was subsequently expressed in a projection-operator form by Lado. ${ }^{6}$ Our purpose here is to propose an alternative generalization which seems to us more closely analogous to the static DCF. We shall refer to this generalization as the time-dependent direct correlation function (TDDCF).

The DCF was originally introduced on an intuitive basis, according to which it was supposed to represent, in essence, the "bare" correlation between a pair of molecules. This interpretation is now known to be approximate. However, there is another interpretation which is completely rigorous: The DCF is simply related to the inverse of the kernel that characterizes the response of the single-molecule distribution function to an infinitesimal single-molecule external field., 7 We are therefore led to consider the possibility of formulating an analogous definition of the TDDCF in terms of the inverse of the kernel which governs the corresponding time-dependent response. This kernel is related to the Van Hove correlation function in the unperturbed fluid by the fluctuation-dissipation theorem of linear response theory. 8,9 We shall see that this approach indeed leads, in a natural way, to an apparently satisfactory definition of the TDDCF.

In the static case, the DCF is useful largely because it exhibits a simpler structure than the total correlation function (or pair distribution function). In particular, the DCF has the universal and remarkably simple asymptotic form $-\beta \theta(12)$, where $\beta=1 / k_{B} T$ and $\theta(12)$ is the pair potential. Similarly, one would expect the TDDCF to be useful if it is in some sense simpler in structure than the Van Hove correlation function. Mean-field considerations provide an indication that this is indeed the case. These considerations suggest that the TDDCF separates into a term which is simply related to $\theta(12)$, and a spatially shorter-ranged remainder. This behavior is confirmed in the Vlasov-Zwanzig approximation.

\section{DEFINITION OF THE TDDCF}

Our development will be presented in a form which applies to molecular as well as atomic fluids, since this entails no additional labor or complexity. Attention is restricted to classical fluids. The single-molecule distribution function $\bar{n}(Q, t)$ is defined as the ensemble average of the molecular quantity

$$
n(Q, t)=\sum_{k} \delta\left(Q-Q_{k}(t)\right),
$$

where $Q$ denotes a dummy set of molecular coordinates, $Q_{k}(t)$ represents the coordinates of molecule $k$ at time $t, \delta(Q)$ is the Dirac delta function in $Q$ space, and the summation extends over all molecules of the system. In the case of atomic fluids, $Q$ simply represents the position vector $\vec{R}$. For molecular fluids, $Q=(\vec{R}, \Omega)$, where $\Omega$ denotes the molecular orientation. For convenience, dummy coordinates $Q_{k}$ will usually be represented by the abbreviated notation $(k)$.

The development is based upon the fluctuationdissipation theorem for the linear response of $\bar{n}(1, t)$ to a single-molecule external potential $\phi_{0}(1, t)$. This theorem takes the for $\mathrm{m}^{8,9}$

$\Delta \bar{n}(1, t)=\int d t^{\prime} \int d(2) K\left(12, t-t^{\prime}\right) \gamma_{0}\left(2, t^{\prime}\right)$,

where $\Delta \bar{n}(1, t)=\bar{n}(1, t)-n_{0}, n_{0}=\langle n(1,0)\rangle_{0}, \gamma_{0}(1, t)$ $=-\beta \phi_{0}(1, t)$,

$$
K(12, t)=-H(t) \frac{\partial}{\partial t}\langle n(2,0) n(1, t)\rangle_{0},
$$

$H(t)$ is zero for $t<0$ and unity otherwise, and the 
angular brackets $\langle\cdots\rangle_{0}$ signify an equilibrium ensemble average in the absence of the external potential. The equilibrium system is assumed to be homogeneous and isotropic, so that $n_{0}$ is a constant independent of $Q_{1}$.

It is usual to discuss time-dependent pair correlations in terms of the Van Hove correlation function $G(12, t)=n_{0}^{-1}\langle n(2,0) n(1, t)\rangle_{0}$. However, we shall find it convenient to utilize a different correlation function which contains the same information. We define the time-dependent total correlation function $\hat{h}(12, t)$ by

$$
\begin{aligned}
n_{0}^{2}[\hat{h}(12, t)+1]= & H(t)\langle n(2,0) n(1, t)\rangle_{0} \\
& +[1-H(t)]\langle n(2,0) n(1,0)\rangle_{0} .
\end{aligned}
$$

This definition has the following consequences: (a) $\hat{h}(12, t)-0$ as $\left|\vec{R}_{1}-\vec{R}_{2}\right| \rightarrow \infty$ or $t \rightarrow \infty$, and (b) $\hat{h}(12, t)=\hat{h}(12)$ for $t \leqslant 0$, where

$$
\hat{h}(12)=h(12)+\delta(12) / n_{0} ;
$$

here $h(12)$ is the static total correlation function, and $\delta(12)=\delta\left(Q_{1}-Q_{2}\right)$. Differentiation of Eq. (4) with respect to $t$ yields

$$
K(12, t)=-n_{0}^{2} \frac{\partial \hat{h}(12, t)}{\partial t},
$$

where use has been made of the fact that $f(t) d H(t) /$ $d t=f(t) \delta(t)=f(0) \delta(t)$. It follows from Eqs. (5) and (6) that

$$
\int d t K(12, t)=n_{0}^{2} \hat{h}(12) .
$$

We now proceed to consider the inverse response. The inverse of Eq. (2) is

$\gamma_{0}(1, t)=\int d t^{\prime} \int d(2) L\left(12, t-t^{\prime}\right) \Delta \bar{n}\left(2, t^{\prime}\right)$,

where $L(12, t)$ is the kernel inverse to $K(12, t)$. In order for Eqs. (2) and (8) to be consistent, we must have

$$
\int d t^{\prime} \int d(2) K\left(12, t-t^{\prime}\right) L\left(23, t^{\prime}\right)=\delta(13) \delta(t) .
$$

Integration of Eq. (9) over $t$ yields

$$
n_{0}^{2} \int d(2) \hat{h}(12) \int d t^{\prime} L\left(23, t^{\prime}\right)=\delta(13),
$$

where Eq. (7) has been used. But the static Ornstein-Zernike equation is just

$$
n_{0}^{2} \int d(2) \hat{h}(12) \hat{c}(23)=-\delta(13)
$$

where

$$
\hat{c}(12)=c(12)-\delta(12) / n_{0},
$$

and $c(12)$ is the static direct correlation function.
Comparison of Eqs. (10) and (11) shows that

$$
\int d t L(12, t)=-\hat{c}(12) \text {. }
$$

According to Eqs. (7) and (13), $-\hat{c}(12)$ is related to the time integral of $L(12, t)$ just as $n_{0}^{2} \hat{h}(12)$ is related to the time integral of $K(12, t)$. It is therefore natural to define a time-dependent direct correlation function $\hat{c}(12, t)$ which is related to $L(12, t)$ in the same way that $-n_{0}^{2} \hat{h}(12, t)$ is related to $K(12, t)$; i.e.,

$$
L(12, t)=\frac{\partial \hat{c}(12, t)}{\partial t} .
$$

To complete the definition, we must specify $\hat{c}(12, t)$ as a function of (12) for some particular value of $t$. This will be done by requiring that $\hat{c}(12, t) \rightarrow 0$ as $t \rightarrow \infty$. Equations (13) and (14) then imply that $\hat{c}(12,-\infty)=\hat{c}(12)$.

Combining Fqs. (6), (9), and (14), we obtain

$n_{0}^{2} \int d t^{\prime} \int d(2) \frac{\partial \hat{h}\left(12, t-t^{\prime}\right)}{\partial t} \frac{\partial \hat{c}\left(23, t^{\prime}\right)}{\partial t^{\prime}}=-\delta(13) \delta(t)$,

which is the time-dependent analog of the OrnsteinZernike equation. It reduces to the static Ornstein-Zernike equation when integrated over $t$.

\section{MEAN-FIELD CONSIDERATIONS}

We now consider the central question of whether $\hat{c}(12, t)$ may be expected to exhibit simple or universal behavior analogous to the universal asymptotic behavior of $c(12)$. We do not attempt a rigorous investigation of this question, but rather provide a suggestive heuristic analysis based on mean-field ideas. This analysis suggests that $\hat{c}(12, t)$ does indeed exhibit such behavior.

If the system were an ideal gas its response behavior could be computed easily, because each molecule would then respond to the external potential $\phi_{0}(1, t)$ as though it were the only molecule present. This simplicity is lost in a dense system; a typical molecule is then subjected not only to $\phi_{0}(1, t)$ but also to the intermolecular potentials of the other molecules. In a mean-field description, one attempts to define a mean or effective field $\phi(1, t)$ which includes both $\phi_{0}(1, t)$ and the average intermolecular potential of the other molecules. The hope is that each molecule will then respond to $\phi(1, t)$ approximately as though it were isolated, so that the system as a whole will respond to $\phi(1, t)$ in approximately the same way that an ideal gas responds to $\phi_{0}(1, t)$. 
The obvious definition of $\phi(1, t)$ would be

$$
\phi(1, t)=\phi_{0}(1, t)+\int d(2) \Delta \bar{n}(2, t) \theta(12),
$$

where $\theta(12)$ is the pair potential. This definition assumes that the molecular interactions propagate instantaneously, and is therefore appropriate only for frequencies sufficiently low that retardation effects are negligible. To deal with higher-frequency response, it would be necessary to replace $\theta(12)$ by a retarded potential $\theta\left(12, t-t^{\prime}\right)$, which would then appear in conjunction with $\Delta \bar{n}\left(2, t^{\prime}\right)$ and a convolution over $t^{\prime}$. These complications will not be considered here.

Unfortunately, Eq. (16) is unsuitable as a general definition of $\phi(1, t)$, because the integral therein does not exist for most pair potentials. (Indeed, it exists unambiguously only for the Coulomb potential.) To make the integral finite, it is evidently necessary to impose some sort of short-range cutoff in the neighborhood of the point $\vec{R}_{2}=\vec{R}_{1}$. Alternatively, we may simply replace $\theta(12)$ in Eq. (16) by any integrable function of (12) that becomes asymptotic to $\theta(12)$ at long range. One such function is $-c(12) / \beta$, and we shall see that this is a particularly natural choice. We therefore adopt it, whereupon Eq. (16) is replaced by

$$
\gamma(1, t)=\gamma_{0}(1, t)+\int d(2) \Delta \bar{n}(2, t) c(12),
$$

where $\gamma(1, t)=-\beta \phi(1, t)$.

The rationale for defining $\phi(1, t)$ in terms of $c$ (12) becomes apparent when Eq. (8) is specialized to the case of static response, in which $\gamma_{0}(1, t)$ and $\Delta \bar{n}(1, t)$ are independent of $t$. With the help of Eqs. (12), (13), and (17), we then obtain

$$
\Delta \bar{n}(1)=n_{0} \gamma(1) \text {. }
$$

But this is precisely the expression for the static response of an ideal gas to an external field $\gamma(1)$. In the static case, therefore, the definition (17) rigorously achieves the mean-field objective of reducing the many-body problem to an ideal-gas problem [assuming, of course, that $c(12)$ is known]. In the absence of other information, it is natural to retain this definition in the dynamical case as well. The rigorous reduction to ideal-gas behavior no longer then obtains, but the corresponding approximation is of potential interest. Indeed, this approximation predicts the same dynamical response as does the Vlasov-Zwanzig equation $^{9,10}$; see Sec. IV.

Combining Eqs. (8) and (17), we obtain

$$
\gamma(1, t)=\int d t^{\prime} \int d(2) L_{s}\left(12, t-t^{\prime}\right) \Delta \bar{n}\left(2, t^{\prime}\right),
$$

where

$$
L_{s}(12, t)=L(12, t)+c(12) \delta(t) .
$$

We note that

$$
\int d t L_{s}(12, t)=\delta(12) / n_{0},
$$

which follows from Eq. (13). Now one would intuitively expect $\Delta \bar{n}(1, t)$ to be more simply and fundamentally related to $\gamma(1, t)$ than to $\gamma_{0}(1, t)$. In particular, the former relation should be more nearly local in space (and also in time if intermolecular retardation effects were included). For this to occur, $L_{s}(12, t)$ must be shorter ranged spatially than $\theta(12)$, and we shall provisionally assume that such is indeed the case. Equations (14) and (20) then imply that $\hat{c}(12, t)$ is of the form

$$
\hat{c}(12, t)=\hat{c}_{s}(12, t)+[1-H(t)] c(12),
$$

where $\hat{c}_{s}(12, t)$ is also shorter ranged spatially than $\theta(12)$ and is determined by

$$
\frac{\partial \hat{c}_{s}(12, t)}{\partial t}=L_{s}(12, t) \text {, }
$$

with $\hat{c}_{s}(12,-\infty)=-\delta(12) / n_{0}$. The presence of the step function $H(t)$ in Eq. (22) is evidently an artifact of the assumption that molecular interactions propagate instantaneously. This function would be smoothed if retardation effects were taken into account.

The above considerations strongly suggest that $\hat{c}(12, t)$ decomposes as shown in Eq. (22), but they are in no sense conclusive. It is therefore of interest to observe that $\hat{c}(12, t)$ does indeed decompose in precisely this way in the well-known Vlasov-Zwanzig approximation, as shown in Sec. IV.

\section{THE VLASOV-ZWANZIG APPROXIMATION}

We now specialize to the case of simple (i.e., atomic) fluids, for which $Q=\vec{R}$ and $n_{0}$ is just the unperturbed number density $\rho$. We define Fourier transforms in space and time by

$$
\begin{aligned}
& \tilde{f}(\overrightarrow{\mathrm{k}})=\int d \overrightarrow{\mathrm{r}} \exp (i \overrightarrow{\mathrm{k}} \cdot \overrightarrow{\mathrm{r}}) f(\overrightarrow{\mathrm{r}}) \\
& \tilde{f}(\overrightarrow{\mathrm{k}}, \omega)=\int d \overrightarrow{\mathrm{r}} \int d t \exp [i(\overrightarrow{\mathrm{k}} \cdot \overrightarrow{\mathrm{r}}-\omega t)] f(\overrightarrow{\mathrm{r}}, t)
\end{aligned}
$$

It is understood that transforms of functions of (12) are obtained by identifying $\vec{r}$ with $\vec{R}_{1}-\vec{R}_{2}$.

The Vlasov-Zwanzig (VZ) equation ${ }^{9,10}$ is a linearized Vlasov equation with the pair potential replaced by $-c(12) / \beta$. This equation determines an approximate expression for the Van Hove correlation function, or, equivalently, for the response kernel $K(12, t)$. The latter is found to $b^{10,11}$

$$
\tilde{K}(\overrightarrow{\mathrm{k}}, \omega)=\frac{\tilde{K}_{0}(\overrightarrow{\mathrm{k}}, \omega)}{1-\tilde{c}(\overrightarrow{\mathrm{k}}) \tilde{K}_{0}(\overrightarrow{\mathrm{k}}, \omega)},
$$


where $\tilde{K}_{0}(\overrightarrow{\mathrm{k}}, \omega)$ is the Fourier transform of the response kernel for an ideal gas. The ideal-gas response is determined by the free-particle Van Hove correlation function

$G_{0}(12, t)=(m \beta / 2 \pi)^{3 / 2}|t|^{-3} \exp \left(-\frac{1}{2} m \beta\left|\vec{R}_{1}-\vec{R}_{2}\right|^{2} / t^{2}\right)$,

together with the fluctuation-dissipation theorem.

To determine $\hat{c}(12, t)$ we must first determine the inverse kernel $L(12, t)$. Fourier transformation of Eq. (9) yields $\tilde{L}(\overrightarrow{\mathrm{k}}, \omega)=1 / \tilde{K}(\overrightarrow{\mathrm{k}}, \omega)$, which combines with Eq. (26) to give

$$
\tilde{L}(\overrightarrow{\mathrm{k}}, \omega)=\tilde{L}_{0}(\overrightarrow{\mathrm{k}}, \omega)-\tilde{c}(\overrightarrow{\mathrm{k}}),
$$

where $\tilde{L}_{0}(\vec{k}, \omega)=1 / \tilde{K}_{0}(\vec{k}, \omega)$ is just the Fourier transform of the inverse response kernel for an ideal gas. The inverse transformation now yields

$$
L(12, t)=L_{0}(12, t)-c(12) \delta(t),
$$

which is of precisely the form given in Eq. (20), with $L_{s}(12, t)=L_{0}(12, t)$. It follows that

$$
\hat{c}(12, t)=\hat{c}_{0}(12, t)+[1-H(t)] c(12),
$$

where $\hat{c}_{0}(12, t)$ is the TDDCF for an ideal gas, which of course is completely determined by $G_{0}(12, t)$ via the fluctuation-dissipation theorem and the relation $\partial \hat{c}_{0}(12, t) / \partial t=L_{0}(12, t)$ [with $\hat{c}_{0}(12,-\infty)$ $=-\delta(12) / \rho]$. Notice that the structure of the $\mathrm{VZ}$ approximation becomes much more transparent when it is expressed in terms of $L(12, t)$ or $\hat{c}(12, t)$, as in Eq. (29) or Eq. (30). A similar structural simplification occurs in the static theory of dielectric polarization in dipolar fluids. ${ }^{12}$

\section{ACKNOWLEDGMENT}

This work was performed under the auspices of the United States Department of Energy.
${ }^{1}$ Physics of Simple Liquids, edited by H. N. V. Temperley, J. S. Rowlinson, and G. S. Rushbrooke (Wiley-Interscience and North-Holland, New York and Amsterdam, 1968).

${ }^{2}$ C. A. Croxton, Liquid State Physics $-A$ Statistical Mechanical Introduction (Cambridge University Press, London, 1974).

${ }^{3}$ H. C. Andersen, Annu. Rev. Phys. Chem. 26, 145 (1975).

${ }^{4}$ J. A. Barker and D. Henderson, Rev. Mod. Phys. $\underline{48}$, 587 (1976).

${ }^{5}$ J. K. Percus and G. J. Yevick, Phys. Fluids 11, 2050
(1968).

${ }^{6}$ F. Lado, Phys. Fluids 13, 1396 (1970).

7J. D. Ramshaw, Mol. Phys. 41, 219 (1980).

${ }^{8}$ R. Kubo, J. Phys. Soc. Jpn. $\underline{12}, 570$ (1957).

${ }^{9}$ N. H. March and M. P. Tosi, Atomic Dynamics in Liquids (Wiley, New York, 1976).

${ }^{10}$ R. Zwanzig, Phys. Rev. 144, 170 (1966).

${ }^{11}$ M. Nelkin and S. Ranganathan, Phys. Rev. 164, 222 (1967).

${ }^{12}$ J. D. Ramshaw, J. Chem. Phys. 73, 5294 (1980), and Refs. 2, 4, and 5 therein. 